\title{
Frequency and characterization of DNA methylation defects in children born SGA
}

\author{
Susanne Bens ${ }^{\star, 1}$, Andrea Haake ${ }^{1}$, Julia Richter ${ }^{1}$, Judith Leohold ${ }^{1}$, Julia Kolarova ${ }^{1}$, Inga Vater ${ }^{1}$, Felix G Riepe ${ }^{2}$, \\ Karin Buiting ${ }^{3}$, Thomas Eggermann ${ }^{4}$, Gabriele Gillessen-Kaesbach ${ }^{5}$, Konrad Platzer ${ }^{5}$, Dirk Prawitt ${ }^{6}$, \\ Almuth Caliebe ${ }^{1}$ and Reiner Siebert ${ }^{1}$
}

Various genes located at imprinted loci and regulated by epigenetic mechanisms are involved in the control of growth and differentiation. The broad phenotypic variability of imprinting disorders suggests that individuals with inborn errors of imprinting might remain undetected among patients born small for gestational age (SGA). We evaluated quantitative DNA methylation analysis at differentially methylated regions (DMRs) of 10 imprinted loci (PLAGL1, IGF2R DMR2, GRB10, H19 DMR, IGF2, MEG3, NDN, SNRPN, NESP, NESPAS) by bisulphite pyrosequencing in 98 patients born SGA and 50 controls. For IGF2R DMR2, methylation patterns of additional 47 parent pairs and one mother (95 individuals) of patients included in the SGA cohort were analyzed. In six out of 98 patients born SGA, we detected DNA methylation changes at single loci. In one child, the diagnosis of upd(14)mat syndrome owing to an epimutation of the MEG3 locus in 14q32 could be established. The remaining five patients showed hypomethylation at $\operatorname{GRB} 10(n=2)$, hypomethylation at the H19 3CTCF-binding site $(n=1)$, hypermethylation at $N D N(n=1)$ and hypermethylation at IGF2 $(n=1)$. IGF2R DMR2 hypermethylation was detected in five patients, six parents of patients in the SGA cohort and two controls. We conclude that aberrant methylation at imprinted loci in children born SGA exists but seems to be rare if known imprinting syndromes are excluded. Further investigations on the physiological variations and the functional consequences of the detected aberrant methylation are necessary before final conclusions on the clinical impact can be drawn.

European Journal of Human Genetics (2013) 21, 838-843; doi:10.1038/ejhg.2012.262; published online 12 December 2012

Keywords: IGF2R; imprinting; methylation; SGA; upd(14)mat

\section{INTRODUCTION}

It is estimated that in humans $>80$ gene loci are subject to imprinting and hence are expressed only from one specific parental allele. ${ }^{1}$ This monoallelic expression is partially achieved by unique epigenetic marks of the maternal and paternal allele, for example, methylation of cytosine residues in the DNA or covalent modification of histone tails. Genes underlying imprinting are not equally distributed in the genome but tend to occur in clusters. Expression of these genes is often regulated by cis-acting elements called imprinting centers (ICs) or imprinting control regions (ICRs) that harbor differential epigenetic marks on the maternal and paternal allele. ${ }^{2}$ The imprinting status of these regulating regions is most frequently determined by DNA methylation analysis of their differentially methylated regions (DMRs). ${ }^{3,4}$

Imprinted regions have been discovered on chromosomes 1, 6, 7, $11,13,14,15$ and 20. Deregulation of these regions is associated with recognizable syndromes depending on the chromosomal region involved.

Two distinct neurogenetic disorders, Angelman syndrome and Prader-Willi syndrome, are both associated with deregulation of imprinted genes in the chromosomal region 15q11q13..$^{5}$ Imprinting defects of the region 11 p15 give rise to the overgrowth syndrome Beckwith-Wiedemann syndrome and the growth retardation disorder Silver-Russell syndrome. ${ }^{6,7}$ Transient neonatal diabetes, characterized by severe intrauterine growth restriction, hyperglycemia and dehydration, is caused by DNA methylation defects of the chromosomal region $6 \mathrm{q} 24 .{ }^{8}$ Uniparental maternal and paternal disomy $14 \mathrm{q} 32$ have been described to be associated with hypomethylation and hypermethylation in $14 \mathrm{q} 32$, respectively. Upd(14)mat, also known as Temple syndrome, is associated with a recognizable phenotype including precocious puberty, short stature, small hands and feet and prominent forehead. ${ }^{9} \mathrm{Upd}(14)$ pat results in a unique phenotype characterized by facial abnormalities, narrow, bell-shaped thorax with coat hanger rib sign, abdominal wall defects and polyhydramnios. Recently different groups identified epimutations in patients with a upd(14)mat and upd(14)pat phenotype. ${ }^{10,11}$

While epimutations represent rare events in Angelman syndrome and Prader-Willi syndrome patients, they are frequently associated with those syndromes characterized by growth abnormalities (eg, Beckwith-Wiedemann syndrome and Silver-Russell syndrome). ${ }^{4-6}$

A key characteristic of genes regulated by epigenetic modification is the involvement in growth and development. Therefore several studies

${ }^{1}$ Institute of Human Genetics, Christian-Albrechts-University Kiel and University Hospital Schleswig-Holstein, Campus Kiel, Kiel, Germany; ${ }^{2}$ Department of Pediatrics, ChristianAlbrechts-University Kiel and University Hospital Schleswig-Holstein, Campus Kiel, Kiel, Germany; ${ }^{3}$ Institute of Human Genetics, University Hospital Essen, Essen, Germany; ${ }^{4}$ Institute of Human Genetics, University Hospital Aachen, Aachen, Germany; ${ }^{5}$ nnstitut für Humangenetik Lübeck, Universität zu Lübeck, Lübeck, Germany; ${ }^{6}$ Section of Molecular Paediatrics, Centre for Paediatrics and Adolescent Medicine, University Medical Centre of the Johannes Gutenberg-University Mainz, Mainz, Germany

${ }^{*}$ Correspondence: Dr S Bens, Christian-Albrechts-University Kiel and University Hospital Schleswig-Holstein, Institute of Human Genetics, Arnold-Heller Str. 3 (Haus 10 ), 24105 Kiel, Germany. Tel: +49 431597 1118; Fax: +49 0431597 1880; E-mail: sbens@medgen.uni-kiel.de

Received 27 July 2012; revised 16 October 2012; accepted 1 November 2012; published online 12 December 2012 
Table 1 Clinical characterization of the 98 children born SGA

\begin{tabular}{|c|c|c|c|c|}
\hline Age groups & Total & $<2$ years & $>2$ years & Data not available \\
\hline Number of included children & 98 & 35 & 63 & 0 \\
\hline Characterization of patient subgroups & Total (n) & Yes (n) & No $(\mathrm{n})$ & Data not available (n) \\
\hline \multicolumn{5}{|l|}{ Prenatal influences } \\
\hline Maternal nicotine consumption & 98 & 21 & 72 & 5 \\
\hline EPH gestosis & 98 & 9 & 56 & 33 \\
\hline Gestational diabetes & 98 & 3 & 47 & 48 \\
\hline Placental insufficiency & 98 & 28 & 43 & 27 \\
\hline \multicolumn{5}{|l|}{ Postnatal outcome } \\
\hline LBW & 98 & 68 & 30 & none \\
\hline SBL & 98 & 73 & 25 & none \\
\hline LBW and SBL & 98 & 58 & 40 & none \\
\hline Lack of catch-up growth (age $>2$ years) & 63 & 50 & 12 & 1 \\
\hline PAH-SDS median (range) & & $-2,38(-1,73--5,43)$ & & \\
\hline $\mathrm{GH}$ therapy (age $>4$ years) & 63 & 37 & 25 & 1 \\
\hline H-SDS before therapy median (range) & & $-3,24(-2,52--5,53)$ & & \\
\hline Developmental delay & 98 & 14 & 82 & 2 \\
\hline
\end{tabular}

Abbreviations: EPH gestosis, edema-proteinuria-hypertension gestosis; GH, growth hormone; H-SDS, body height standard deviation; LBW, low birth weight; PAH-SDS, parent adjusted height standard deviation (defined as body height SDS - target height SDS); SBL, short birth length. Lack of catch-up growth is defined as H-SDS $<-2.5$ and PAH-SDS $<-1$.

aimed to investigate whether imprinting defects are a frequent underlying cause for intrauterine growth restriction. Most frequently the region $11 \mathrm{p} 15$ has been in focus, as hypomethylation of the imprinting control region 1 leads to reduced IGF2 expression and hence growth retardation. ${ }^{6}$ Results have been very heterogeneous with most studies analyzing chorionic villi or placenta tissue. ${ }^{12-15}$ Nevertheless, placental tissue displays an intermediate distribution of methylation as compared, for example, with embryonal epithelial cells. ${ }^{16,17}$ Moreover, there is greater epigenetic variation in extraembryonic than in embryonic tissue. ${ }^{18}$ However, it is intriguing to postulate that in some children born small for gestational age (SGA) the underlying cause is a yet unrecognized imprinting disorder. Turner et al ${ }^{12}$ detected hypermethylation in the IGF2R DMR2 to be enriched among patients with growth restriction. They hypothesized that hypermethylation of IGF2R DMR2 results in increased expression of IGF2R and lower circulating levels of IGF2, eventually explaining growth restriction. ${ }^{12}$

In order to gain more insight in the underlying causes of growth restriction, we here performed a screening study of 10 imprinted loci, including those associated with fetal growth restriction, on peripheral blood of a large cohort of 98 children born SGA.

\section{MATERIALS AND METHODS}

The study has been approved by the Institutional Review Board of the Medical Faculty of the Christian Albrechts University Kiel (No B305/08).

\section{Patient and control samples}

We recruited 98 children born SGA (64 boys and 34 girls) among the cooperating centers of the BMBF consortium. Inclusion criteria for SGA were defined as birth length and/or birth weight under -2 SD for gestational age, according to the reference data published by Niklasson et $a l^{19}$, and informed consent of the participating families. There were no exclusion criteria. Dysmorphisms, as well as clinical details concerning pregnancy, and postnatal development were documented if available (Table 1). We divided the included children according to age in two subgroups with a cutoff at the age of 2 years, because catch up growth has been reported to be completed by then. $^{20,21}$ The group of children aged younger than 2 years comprised 35 children. Median age in this group was 6 days (range: 1 day-19 months). In the group of children aged over 2 years, median age was 9.5 years (range: 4-18 years) (compare Table 1). A certain bias toward children being more severely affected by SGA cannot be excluded, as those children are more likely to come to medical attention.

The control cohort comprised anonymized samples of 50 reportedly healthy probands of Caucasian ancestry. Each of the cooperating centers contributed 10 control samples ( 5 males, 5 females). For IGF2R DMR2 methylation, we additionally studied 95 parents of patients from the SGA cohort (47 parent pairs and one single mother).

\section{Bisulphite pyrosequencing}

Pyrosequencing assays have been designed using the PyroMark Assay Design Software (Version 1.0 and 2.0, Qiagen, Hilden, Germany). For PCR and primer conditions, see Supplementary Table S1.

We established 11 assays based on literature review $^{22-29}$, as shown in Supplementary Table S2. We failed to establish a reliable bisulphite pyrosequencing assay for the KvDMR/IC2 in 11p15.

Genomic DNA was extracted from whole peripheral blood and bisulphite converted using the EpiTect Bisulfite Conversion Kit (Qiagen, Hilden, Germany). PCR product sizes were verified by gel electrophoresis. Singlestrands were prepared using the Vacuum Prep Tool (Biotage, Uppsala, Sweden), followed by a denaturation step at $85^{\circ} \mathrm{C}$ for $2 \mathrm{~min}$ and final sequencing primer hybridization. Pyrosequencing was performed using the Pyrosequencer ID and the DNA methylation analysis software Pyro Q-CpG 1.0.9 (Biotage), which was also used to quantify the ratio $\mathrm{T}: \mathrm{C}(\mathrm{mC}: \mathrm{C})$ at the analyzed $\mathrm{CpG}$ sites. Assays were validated using a commercial in vitro methylated DNA as positive control (Millipore, Hilden, Germany) and pooled DNA from 20 healthy donors (10 males, 10 females).

\section{Analysis of bisulphite pyrosequencing data}

In order to establish a method to detect hypo- and hypermethylated samples on the basis of methylation raw data, we first aimed to define a 'normal' methylation range for each pyrosequencing assay on the basis of results from the control cohort. For details on how the 'normal methylation range' was obtained for each assay, refer to Supplementary Table S3. Next, we compared the results of the patient samples with the 'normal methylation range' obtained 


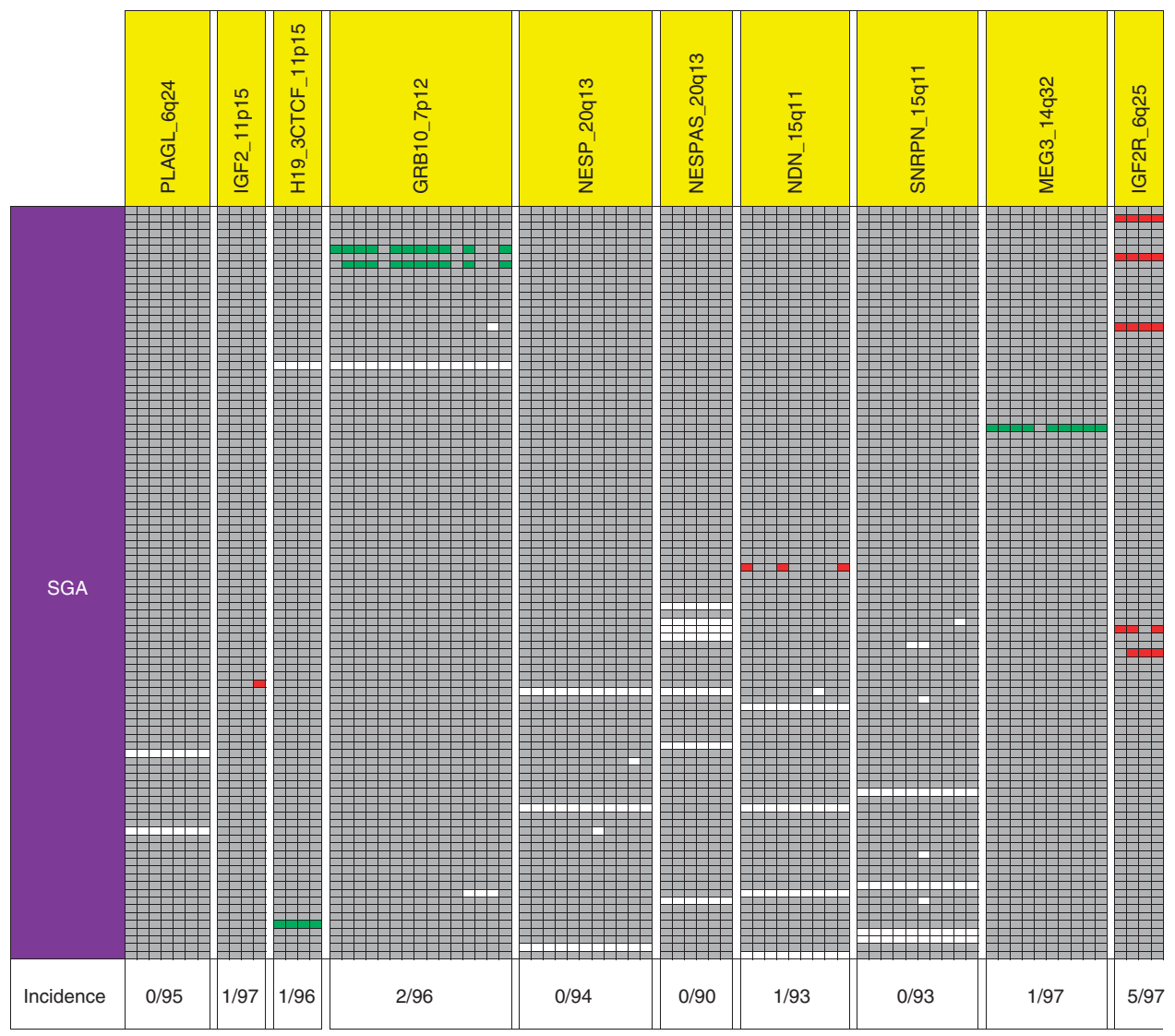

Figure 1 Pyrosequencing results of the analyzed patients born SGA. Each column represents one CpG position; each line represents one studied patient sample. Mean sample methylation values ('mean(locus)') are displayed in the outer right column of each assay. If the 'mean(locus)' of a sample exceeded the assay-specific normal methylation range (NMR: mean(locus/controls) $\pm 3 S D$ ), the sample was called 'hypermethylated' (displayed in red); if it was below the NMR, the sample was called 'hypomethylated' (displayed in green). 'mean(locus)' values within the NMR were called normal methylated (displayed in gray). For such 'hyper-' and 'hypomethylated' loci, we determined for each single CpG in this locus whether its methylation level was outside the mean methylation level of the same CpG in the 50 controls \pm 3 SD. Results for the SGA study cohort revealed single-locus methylation defects in six patients (hypomethylation at MEG3 $(n=1)$, hypomethylation at GRB1O $(n=2)$, hypomethylation at H19_3CTCF $(n=1)$, hypermethylation at NDN $(n=1)$ and hypermethylation at IGF2 $(n=1)$ ). In addition, five patients displayed IGF2R DMR2 hypermethylation. Failed analysis is displayed white.

for all analyzed loci. If the mean methylation value for a given locus exceeded the defined assay specific 'normal range' in a patient sample, the overall methylation result for that locus was called 'hypermethylated'. If the mean methylation value for a given locus was below the assay specific 'normal range' in a patient sample, the overall methylation result for this locus was called 'hypomethylated'. If the overall methylation result for a locus was defined as aberrant in a patient sample, the results of each $\mathrm{CpG}$ position were evaluated separately by comparing the methylation value with the 'normal methylation range' of the corresponding $\mathrm{CPG}$ position.

\footnotetext{
Methylation-specific PCR of the MEG3 promoter and the DLK1/ MEG3 differentially methylated intergenic region

For the MEG3 locus on chromosome 14q32, a combined (duplex) methylation -specific PCR analysis was carried out as described by Dietz et al..$^{30}$

Methylation-specific PCR for the differentially methylated intergenic region between DLK1 and MEG3 was performed by using FAM-labeled specific primers for the methylated paternal and the unmethylated maternal allele. For primer sequences, refer to Supplementary Table S1. PCR was carried out as follows: $95^{\circ} \mathrm{C}$ for $10 \mathrm{~min}, 35$ cycles of $95^{\circ} \mathrm{C}$ for $30 \mathrm{~s}, 58^{\circ} \mathrm{C}$ for $30 \mathrm{~s}, 72^{\circ} \mathrm{C}$ for $30 \mathrm{~s}$ and $30 \mathrm{~min}$ at $72^{\circ} \mathrm{C}$. The PCR products were visualized on a $2 \%$ agarose gel and then subjected to fragment length analysis on an ABI 3100 Genetic Analyzer (Applied Biosystems, Foster City, CA, USA) using the GeneScan software (Applied Biosystems).
}

\section{Methylation-specific (MS) MLPA analysis for the chromosomal region $11 \mathrm{p} 15$}

MS-MLPA analysis was performed with the Salsa MLPA Kit ME030-B1, according to the manufacturer's protocol (MRC Holland, Amsterdam, The Netherlands).

\section{Microarray analysis}

The Genome-wide human SNP array 6.0 (Affymetrix, Santa Clara, CA, USA) was used according to the protocol provided by the manufacturer. Microarrays were washed and stained with the Fluidics Station 450 (Affymetrix), scanned with the GeneChip Scanner 3000 (Affymetrix) and analyzed with the Genotyping Console software version 4.0 (Affymetrix).

\section{Microsatellite analysis for chromosome 7 and 14}

PCR was carried out using one fluorescent-labeled primer in the PCR reaction. Fragment analysis and interpretation were performed with the ABI 3100 Genetic Analyzer, the GeneMarker Software (Softgenetics) and the GeneMapper 3.5 Software (Applied Biosystems), according to the standard procedures. The following microsatellite markers were used on chromosome 7 flanking GRB10 on both sides: D7S1813, D7S3050, D7S2431, D7S1812, D7S2420, D7S2544, D7S649, D7S2422, D7S813, D7S1818 and D7S674. For chromosome 14 , the following markers, located throughout chromosome 14, were used: 
Table 2 Phenotype of patients with single-locus methylation defect among the SGA cohort

\begin{tabular}{|c|c|c|c|}
\hline $\begin{array}{l}\text { Affected } \\
\text { locus }\end{array}$ & Methylation detail & Age (gender) & Clinical details \\
\hline MEG3 & LoM (12\%), 8/9 & 5 years $(F)$ & $\begin{array}{l}\text { Short stature, precocious pub- } \\
\text { erty, developmental delay, small } \\
\text { hands and feet, prominent } \\
\text { forehead }\end{array}$ \\
\hline GRB10 & LoM (20\%), 10/14 & 17 years $(\mathrm{M})$ & $\begin{array}{l}\text { Short stature, learning } \\
\text { difficulties, overfolded ear } \\
\text { helices, hexadactyly left hand }\end{array}$ \\
\hline GRB10 & LoM (20\%), 9/14 & 13 years $(\mathrm{M})$ & $\begin{array}{l}\text { Short stature, no dysmorphic } \\
\text { features }\end{array}$ \\
\hline H19_3CTCF & LoM (4\%), 3/3 & 12 years $(F)$ & $\begin{array}{l}\text { Clinodactyly, mother with dia- } \\
\text { betes mellitus type I }\end{array}$ \\
\hline NDN & GoM (63\%), 2/8 & 7 years $(M)$ & $\begin{array}{l}\text { DZ twin pregnancy, umbilical } \\
\text { hernia, ichthyosis, flat philtrum, } \\
\text { epicanthus, hypertelorism, thin } \\
\text { lip, camptodactyly }\end{array}$ \\
\hline IGF2 & GoM $(62 \%), 0 / 3$ & 3 days $(F)$ & $\begin{array}{l}\text { Mother with two early } \\
\text { miscarriages, one healthy } \\
\text { sibling }\end{array}$ \\
\hline
\end{tabular}

Abbreviations: F, female; M, male; LoM, loss of methylation/hypomethylation; GoM, gain of methylation/hypermethylation.

aMethylation status (mean sample DNA methylation in \%) and number of affected CpG positions ( $($ (affected) $/ n$ (analyzed)).

D14S597, D14S290, D14S81, D14S267, D14S250, D14S78, D14S1006 and D14S1010.

\section{RESULTS}

In the present study, we analyzed 98 patients born SGA. While for several probands dysmorphic features were noted, none displayed the combined characteristic features of Silver-Russell syndrome. In 40 children born SGA, the condition was unexplained. In the remaining children prenatal factors potentially contributing to growth retardation could not be excluded (Table 1). While one could anticipate that intrauterine growth restriction might have contributed to the SGA condition in those children with placental insufficiency, we are not able to prove this, as we have no systematic information on any prenatal measurements for the included children. In the group of children aged over 2 years, 50 out of 63 patients had lack of catch up growth and permanent short stature. Thirty-seven patients received growth hormone treatment. Mental retardation was reported for 14 patients (Table 1).

DNA methylation of the included 98 children born SGA were analyzed at the imprinted loci PLAGL1, IGF2R DMR2, GRB10, IGF2, H19_3CTCF, H19_6CTCF, MEG3, NDN, SNRPN, NESP and NESPAS. Because of high variability of DNA methylation at H19_6CTCF among the control cohort, we were statistically not able to detect hypomethylation (NMR: 0-54\%). For alternative evaluation, the H19_3CTCF assay was used (NMR: 25-51\%).

One sample failed in more than two assays and was therefore excluded from the evaluation. Among the SGA cohort, we detected aberrant methylation values within one of the 10 assays in 6 out of the remaining 97 patients (Figure 1). Five of them were detected in the cohort of children older than 2 years. Clinical details for these patients are described in Table 2. Among the 6 patients with aberrant methylation values five presented at birth with both low body weight and short body length. Two of the six patients have documented failed catch up growth.
In one case, MEG3 DMR hypomethylation (mean methylation value $12 \%$ ) was detected. Methylation-specific PCR of the MEG3 promoter and the DLK1/MEG3 differentially methylated intergenic region (IG-DMR) revealed absence of the methylated allele, thereby confirming the pyrosequencing results and leading to the tentative diagnosis of upd(14)mat syndrome. Further diagnostic workup by microsatellite and array analysis revealed biparental inheritance and no genomic imbalances in the chromosomal region $14 \mathrm{q} 32$ containing the MEG3 gene. The diagnosis of upd(14)mat syndrome caused by an epimutation was established.

One case with hypomethylation in H19_3CTCF (mean methylation value 4\%) was detected. MLPA analysis of the chromosomal region 11 p15 showed normal results; however, the methylationsensitive probes of the MLPA kit and the pyrosequencing assay are $\sim 3 \mathrm{~kb}$ apart.

Furthermore, hypomethylation of GRB10 was detected in two patients. Mean methylation values were $20 \%$ in both cases, while the assay-specific NMR for GRB10 ranges from $27-46 \%$. These results could represent a mosaic hypomethylation of GRB10 comparable to the mosaic findings often reported for Silver-Russell and BeckwithWiedemann patients with methylation changes in 11p15. Further workup by microsatellite analysis revealed in both cases biparental inheritance for the chromosomal region $7 \mathrm{p} 12$ containing the GRB10 gene. The remaining two cases showed isolated hypermethylation at the NDN (mean methylation value of 63\%) and IGF2 locus (mean methylation value of 62\%). The latter finding was the only aberrant methylation value detected in the subgroup of children younger than 2 years.

Moreover, independent from the statistical approach used, 5 patients ( 4 out of the subgroup of children older than 2 years and one child younger than 2 years) with IGF2R DMR2 hypermethylation were detected in the patient cohort (Figure 1, Table 3). Two presented at birth with low body weight, one with short body length and two with low body weight combined with short body length. Three out of the four affected patients older than 2 years have failed catch up growth as documented. In the control cohort, 2 and additional 3 out of 50 probands displayed hypermethylation, depending on the statistical approach used for defining hypermethylation cutoff, respectively. To study the impact of hypermethylation at the IGF2R locus on fetal growth of offspring, we additionally analyzed the methylation status of IGF2R in 47 parent pairs and one single mother of the SGA cohort. We detected six individuals (three fathers, three mothers) with IGF2R DMR2 hypermethylation. Among the studied trios, we found two families, in which IGF2R DMR2 hypermethylation affected more than one individual (for details see Figure 2).

\section{DISCUSSION}

In this study, we aimed to investigate whether as yet unrecognized imprinting defects are a frequent cause in children born SGA. We here applied bisulphite pyrosequencing, because the advantages of this technique are the fast and quantitative methylation analysis of several neighboring $\mathrm{CpG}$ positions, which is the reason for its widespread use in high-throughput studies. ${ }^{31}$ While some authors claim excellent accuracy, ${ }^{32}$ in our experience from extensive dilution series, the detection of methylation status is not always linear, for example because of selective amplification of one parental allele, and highly dependent on the assay in use. We applied high-quality requirements for the establishment of new pyrosequencing assays. However, we were not able to establish a reliable pyrosequencing assay for the KvDMR/IC2 in 11p15.5 that would satisfy our quality criteria. Bearing in mind that aberrant methylation patterns at the KvDMR/IC2 in 
Table 3 Phenotype of patients with IGF2R DMR2 hypermethylation among the SGA cohort

\begin{tabular}{|c|c|c|c|}
\hline $\begin{array}{l}\text { Affected } \\
\text { locus }\end{array}$ & $\begin{array}{l}\text { Methylation } \\
\text { detail }\end{array}$ & Age (gender) & Clinical details \\
\hline IGF2R & GoM $(82 \%), 3 / 3$ & 14 years $(\mathrm{M})$ & $\begin{array}{l}\text { Short stature, large ears, mild } \\
\text { clinodactyly }\end{array}$ \\
\hline IGF2R & GoM $(82 \%), 3 / 3$ & 9 years $(\mathrm{M})$ & $\begin{array}{l}\text { Flat midface, protruding ears, clino- } \\
\text { dactyly, inguinal hernia }\end{array}$ \\
\hline IGF2R & GoM (92\%), 2/3 & 4 days $(F)$ & $\begin{array}{l}\text { Short stature, thin lips, anteverted } \\
\text { nares }\end{array}$ \\
\hline IGF2R & GoM (84\%), 3/3 & 9 years $(\mathrm{M})$ & $\begin{array}{l}\text { Adipositas, disproportionate short } \\
\text { stature, round face, prominent ear } \\
\text { lobes, anteverted nares, prominent } \\
\text { forehead }^{\text {b }}\end{array}$ \\
\hline IGF2R & GoM (85\%), 2/3 & 4.5 years $(F)$ & Bladder exstrophy, inguinal hernia \\
\hline
\end{tabular}

Abbreviations: $F$, female, GoM, gain of methylation/hypermethylation; $M$, male.

a Methylation status (mean sample DNA methylation in \%) and number of affected CpG positions ( $n$ (affected) $/ n$ (analyzed)).

${ }^{\mathrm{b}} \mathrm{A}$ maternally transmitted IGF1 missense mutation of unknown significance was reported.

11 p15.5 are most frequently associated with overgrowth, we think that this assay is dispensable in the screening set. Involvement of KvDMR/ IC2 in growth restriction was only reported in the context of multiple methylation defects. ${ }^{33}$

With the statistical approach applied here for the definition of 'hypermethylation' and 'hypomethylation', we would anticipate to identify $99.7 \%$ of cases showing normal methylation patterns correctly. Hence, taking into account that nearly 1000 analyses were evaluated (10 loci, 98 patients), we would expect roughly three falsepositive results, that is, samples with normal methylation patterns diagnosed as 'hyper-' or 'hypomethylated'. This is only a rough estimation, as our findings show methylation levels of IGF2R, as well as NDN, and SNRPN to be not or borderline equally distributed.

In this context, it is remarkable, that for IGF2 none of three and for NDN only 2/8 single CpGs within the locus diagnosed as aberrantly methylated, actually showed significant methylation changes (see Figure 1). Moreover, in the instance of IGF2-DMR0 hyermethylation, no aberrant methylation at the H19 locus was detected. The latter is normally regulating the IGF2 secondary DMRs in a hierarchical fashion. As in addition, most hitherto characterized epimutations usually affect the majority of neighboring CpG positions in a regulatory DMR we tend to conclude, that these both instances are likely false positives.

By analyzing the peripheral blood of 98 children born SGA with no further symptoms of Silver-Russell syndrome, we found six children to display single-locus methylation defects. None of the children displayed multiple methylation defects. In addition, we have not detected hypomethylation of the H19_3CTCF-binding site to represent a frequent finding in children born SGA.

In one of the six children with single-locus methylation defect, the diagnosis of upd(14)mat syndrome owing to an epimutation was established. Clinical findings for this child are well consistent with the newly established diagnosis: the girl is developmentally delayed, presents with short stature, developed a precocious puberty, has small hands and feet as well as a prominent forehead.

In the child with H19_3CTCF hypomethylation, we repeatedly detected absent methylation values, while the H19_6CTCF binding site revealed normal methylation patterns in MS-MLPA. The girl has clinodactyly of the fifth finger on both hands. She has no further dysmorphic signs. As outlined above, we detected high variability in

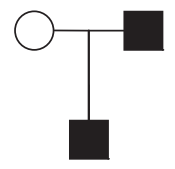

family 1

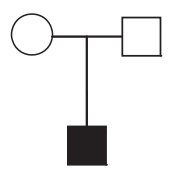

family 7

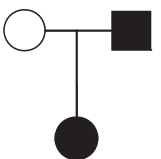

family 2

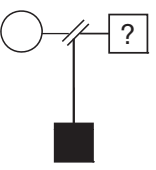

family 8

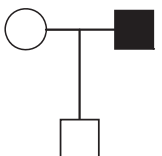

family 3

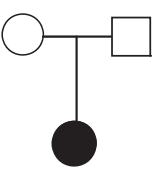

family 9

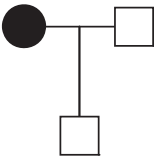

family 4-6

\section{IGF2R DMR2 hypermethylation}

Figure 2 Pedigrees of the trios in which either the child or a parent displays IGF2R DMR2 hypermethylation.

the H19DMR/IC1 locus, especially in the H19_6CTCF assay, among controls. A similar observation was made by Coolen et $a^{34}$, who analyzed amongst other loci the H19 DMR in MZ and DZ twins.

Extensive studies on CTCF-binding sites in that region concluded that especially the 6CTCF-binding site acts as a key regulator domain for H19/IGF2 expression. ${ }^{35}$ Hence it is possible that the impact on phenotype in a patient with hypomethylation at the H19 3CTCF-binding site is less severe compared with patients with hypomethylation at the H19 6CTCF-binding site and the classical phenotype of Silver-Russell syndrome.

Two children were found to display hypomethylation for GRB10, which encodes the growth factor receptor-bound protein 10 . While one of the two patients has no dysmorphic signs, the other patient has hexadactyly of his left hand and overfolded ear helices. For the latter patient, learning difficulties are reported. Both patients are short of stature and receive growth hormone treatment. While the patient without dysmorphic features shows an improvement of body height of $+1.5 \mathrm{SD}$ since the beginning of treatment, the other patient has no significant benefit in relative height since the introduction of growth hormone treatment. We confirmed biparental inheritance of the chromosomal region 7p12, containing the GRB10 gene by microsatellite analysis in both cases. Animal studies showed that the mouse homolog Grb10 is subject to imprinting and is expressed predominantly from the maternal allele. Disruption of the maternal allele leads to overgrowth of placenta and embryo with the mutant mice being $\sim 30 \%$ larger than the wild-type mice. ${ }^{36}$ Moreover GRB10 has long been proposed as responsible candidate gene for Silver-Russell syndrome in maternal uniparental disomy for chromosome 7 and maternal duplication of the chromosomal region $7 \mathrm{p} 11.2-\mathrm{p} 12 . .^{37,38}$ However, exclusion of causative mutations in GRB10 in patients with Silver-Russell syndrome and the identification of patients with segmental maternal uniparental disomy restricted to the long arm of chromosome 7 make a relevant role of GRB10 in patients with SilverRussell syndrome unlikely. ${ }^{39,40}$ Our observation adds new aspects in this complex field: whereas GRB10 seems to have a minor role in Silver-Russell syndrome, dysregulation might be relevant for the milder phenotype SGA.

In contrast to previous studies, we have not found IGF2R DMR2 hypermethylation to be enriched among children born SGA. ${ }^{12}$ Turner et $a^{12}$ detected significant enrichment of IGF2R hypermethylation in $7 / 79$ patients with growth restrictions versus $3 / 267$ controls. In this study, we detected IGF2R hypermethylation in 5/98 children born SGA. However, in line with recent publications, we detected IGF2R 
DMR2 hypermethylation repeatedly and in similar frequency (2 and $5 / 50$ samples, depending on the strategy of analyzing pyrosequencing data) within our control cohort. ${ }^{12,41}$ The lack of phenotypic correlation in our and previous studies leads to the assumption that IGF2R DMR2 hypermethylation most likely represents an epigenetic polymorphism, a Mendelian inheritance cannot be excluded from our observations.

We conclude that aberrant methylation at imprinted loci in children born SGA exists, but seems to be rare if known imprinting syndromes are excluded. Further investigations on the physiological variations and the functional consequences of the detected aberrant methylation are necessary before final conclusions on the clinical impact can be drawn.

\section{CONFLICT OF INTEREST}

The authors declare no conflict of interest.

\section{ACKNOWLEDGEMENTS}

We thank Ute Jacobsen, Gabriele Riesen, Claudia Becher and Christina Lich for their excellent technical assistance. The research leading to these results has received funding from the German national BMBF (Ministry of Education and Science)-funded consortium 'Diseases caused by imprinting defects: clinical spectrum and pathogenetic mechanisms' (FKZ: 01GM0886 and 01GM1114).

1 Glaser RL, Ramsay JP, Morison IM: The imprinted gene and parental-of-origin effect database now includes parental origin of de novo mutations. Nucleic Acids Res 2006; 34: D29-D31.

2 Buiting K, Saitoh S, Gross S et al: Inherited microdeletions in the Angelman and Prader-Willi syndromes define an imprinting centre on human chromosome 15. Nat Genet 1995; 9: 395-400.

3 Ammerpohl O, Siebert R: How to analyse epigenetic marks. Pediatr Endocriol Rev 2011; 9: 511-514.

4 Horsthemke B: Epimutations in human disease. Curr Top Microbiol Immunol 2006, 310: 45-59.

5 Buiting K: Prader-Willi syndrome and Angelman syndrome. Am J Med Genet C Semin Med Genet 2010; 154C: 365-376.

6 Gicquel C, Rossignol S, Cabrol S et al: Epimutation of the telomeric imprinting center region on chromosome 11q15 in Silver-Russell syndrome. Nat Genet 2005; 37: 1003-1007.

7 Choufani S, Shuman C, Weksberg R: Beckwith-Wiedemann syndrome. Am J Med Genet C Semin Med Genet 2010; 154C: 343-354.

8 Gardner RJ, Mackay DJG, Mungall AJ et al: An imprinted locus associated with transient neonatal diabetes mellitus. Hum Mol Genet 2000; 9: 589-596.

9 Temple IK, Shrubb V, Lever M, Bullman H, Mackay DJG: Isolated imprinting mutation of the DLK1/GTL2 locus associated with a clinical presentation of maternal uniparental disomy of chromosome 14. J Med Genet 2007; 44: 637-640.

10 Buiting K, Kanber D, Martin-Subero JI et al: Clinical features of maternal uniparental disomy 14 in patients with an epimutation and a deletion of the imprinted DLK1/GTL2 gene cluster. Hum Mutat 2008; 29: 1141-1146.

11 Kagami M, Sekita Y, Nishimura G et al: Deletions and epimutations affecting the human 14q32.2 imprinted region in individuals with paternal and maternal upd(14)like phenotypes. Nat Genet 2008; 40: 237-242.

12 Turner CLS, Mackay DM, Callaway J et al: Methylation analysis of 79 patients with growth restriction reveals novel patterns of methylation change at imprinted loci. Eur J Hum Genet 2010; 18: 648-655.

13 Guo L, Choufani S, Ferreira J et al: Altered gene expression and methylation of the human chromosome 11 imprinted region in small for gestational age (SGA) placentae. Dev Biol 2008; 320: 79-91.

14 Bourque DK, Avila L, Penaherrera M, von Dadelszen P, Robinson WP: Decreased placental methylation at the H19/IGF2 imprinting control region is associated with normotensive intrauterine growth restriction but not preeclampsia. Placenta 2010; 31: 197-202.

15 Tabano S, Colapeitro P, Cetin I et al: Epigenetic modulation of the IGF2/H19 imprinted domain in human embryonic and extra-embryonic compartments and its possible role in fetal growth restriction. Epigenetics 2010; 5: 313-324.
16 Chu T, Handley D, Bruce K, Surti U, Hogge WA, Peters DG: Structural and regulatory characterization of the placental epigenome at its maternal interface. PLOS ONE 2011; 6: e14723.

17 Eckmann-Scholz C, Bens S, Kolarova J et al: DNA-methylation profiling of fetal tissues reveals marked epigenetic differences between chorionic and amniotic samples. PLoS ONE 2012; 7: e39014.

18 Turan N, Katari S, Gerson LF et al: Inter- and intra-individual variation in allele-specific DNA methylation and gene expression in children conceived using assisted reproductive technology. PLoS Genet 2010; 6: e1001033.

19 Niklasson A, Albertsson-Wikland K: Continous growth reference from 24th week of gestation to 24 months by gender. BMC Pediatr 2008; 29: 8

20 Hokken-Koelega AC, De Ridder MA, Lemmen RJ, Den Hartog H, De Muinck KeizerSchrama SM, Drop SL: Children born small for gestational age: do they catch up? Pediatr Res 1995; 38: 267-271.

21 Karlberg J, Albertsson-Wikland K: Growth in full-term small-for-gestational-age infants: from birth to final height. Pediatr Res 1995; 38: 733-739.

22 Arima T, Drewell RA, Arney $\mathrm{KL}$ et al: A conserved imprinting control region at the HYMAI/ZAC domain is implicated in transient neonatal diabetes mellitus. Hum $\mathrm{Mol}$ Genet 2001; 10: 1475-1483.

23 Hikichi T, Kohda T, Kaneko-Ishino T, Ishino F: Imprinting regulation of the murine Meg1/Grb10 and human GRB10 genes; roles of brain-specific promoters and mousespecific CTCF-binding sites. Nucleic Acids Res 2003; 31: 1398-1406.

24 Liu Z, Wang Z, Li Y et al: Association of genomic instability, and the methylation status of imprinted genes and mismatch-repair genes, with neural tube defects. Eur J Hum Genet 2012; 20: 516-520.

25 Takai D, Gonzales FA, Tsai YC, Thayer MJ, Jones PA: Large scale mapping of methycytosines in CTCF-binding sites in the human $\mathrm{H} 19$ promoter and aberrant hypomethylation in human bladder cancer. Hum Mol Genet 2001; 10: 2619-2626.

26 Liu J, Nealon JG, Weinstein LS: Distinct patterns of abnormal GNAS imprinting in familial and sporadic pseudohypoparathyroidism type IB. Hum Mol Genet 2005; 14: 95-102.

27 Lau JCY, Hanel ML, Wevrick R: Tissue-specific and imprinted epigenetic modifications of the human NDN gene. Nucleic Acids Res 2004; 32: 3376-3382.

28 Zeschnigk M, Schmitz B, Dittrich B, Buiting K, Horsthemke B, Doerfler W: Imprinted segments in the human genome: different DNA methylation patterns in the PraderWilli/Angelman syndrome region as determined by the genomic sequencing method. Hum Mol Genet 1997; 6: 387-395.

29 Smrzka OW, Fae I, Stöger R et al: Conservation of a maternal-specific methylation signal at the human IGF2R locus. Hum Mol Genet 1995; 4: 1945-1952.

30 Dietz LG, Wylie AA, Rauen KA, Murphy SK, Jirtle RL, Cotter PD: Exclusion of maternal uniparental disomy of chromosome 14 in patients referred for Prader-Willi syndrome using a multiplex methylation polymerase chain reaction assay. J Med Genet 2003; 40: e46.

31 Ammerpohl O, Martin-Subero JI, Richter J, Vater I, Siebert R: Hunting for the 5th base: Techniques for analyzing DNA methylation. Biochim Biophys Acta 2009; 1790: 847-862.

32 Potapova A, Albat C, Hasemeier B et al: Systematic cross-validation of 454 sequencing and pyrosequencing for the exact quantification of DNA methylation patterns with single CpG resolution. BMC Biotechnol 2011; 11: 6 .

33 Begemann M, Spengler S, Kanber D et al: Silver-Russell patients showing a broad range of ICR1 and ICR2 hypomethylation in different tissues. Clin Genet 2011; 80: 83-88.

34 Coolen MW, Statham AL, Qu W et al: Impact of the genome on the epigenome is manifested in DNA methylation patterns of imprinted regions in monozygotic and dizygotic twins. PLoS ONE 2011; 6: e25590.

35 Takai D, Gonzales FA, Tsai YC, Thayer MJ, Jones PA: Large scale mapping of the methylcytosines in CTCF-binding sites in the human H19 promoter and aberrant hypomethylation in human bladder cancer. Hum Mol Genet 2001; 10: 2619-2626.

36 Charalambous M, Smith FM, Bennett WR, Crew TE, Mackenzie F, Ward A: Disruption of the imprinted Grb10 gene leads to disproportionate overgrowth by an Igf2independent mechanism. Proc Natl Acad Sci USA 2003; 100: 8292-8297.

37 Joyce CA, Sharp A, Walker JM, Bullman H, Temple IK: Duplication of 7p12.1-p13, including GRB10 and IGFBP1, in a mother and daughter with features of SilverRussell syndrome. Hum Genet 1999; 105: 273-280.

38 Monk D, Wakeling EL, Proud V et al: Duplication of 7q11.2-p13, including GRB10, in Silver-Russell syndrome. Am J Hum Genet 2000; 66: 36-46.

39 Eggermann T, Schönherr N, Jäger S et al: Segmental maternal UPD(7q) in SilverRussell syndrome. Clin Genet 2008; 74: 486-489.

40 Hitchins MP, Monk D, Bell GM et al: Maternal repression of the human GRB10 gene in the developing central nervous system; evaluation of the role for GRB10 in SilverRussell syndrome. Eur J Hum Genet 2001; 9: 82-90.

41 Poole RL, Baple E, Crolla JA, Temple IK, Mackay DJG: Inverstigation of 90 patients referred for molecular cytogenetic analysis using aCGH unconvers previously unsuspected anomalies of imprinting. Am J Med Genet A 2010; 152A: 1990-1993.

Supplementary Information accompanies the paper on European Journal of Human Genetics website (http://www.nature.com/ejhg) 